\title{
Las respuestas de la Unión Europea a las transiciones inversas en Cuba y Venezuela
}

\author{
Anuario Latinoamericano \\ Ciencias Políticas \\ y Relaciones Internacionales \\ vol. 9, 2020 \\ pp. $89-112$
}

\section{The European Union's Responses to the Inverse Transitions in Cuba and Venezuela}

\author{
Anna Ayuso* \\ BARCELONA CENTER FOR INTERNATIONAL AFFAIRS \\ BARCELONA, ESPAÑA \\ $\triangle$ aayuso@cidob.org \\ http//orcid.org/0000-0003-1301-851X \\ Susanne Gratius ${ }^{\star *}$ \\ UNIVERSIDAD AUTÓNOMA DE MADRID \\ MADRID, ESPAÑA \\ $\triangle$ susanne.gratius@uam.es \\ http://orcid.org/0000-0002-4005-4498
}

DOI: 10.17951/al.2020.9.89-112

\section{RESUMEN}

Frente a los regímenes autoritarios de Cuba y Venezuela, la Unión Europea (UE) ha dado respuestas distintas: diálogo político y cooperación en el primer caso, sanciones selectivas y ruptura diplomática en el segundo. El artículo indaga a qué se deben estas diferencias, a pesar de servir al mismo objetivo: promover la democracia y los derechos humanos. Una posible explicación serían las transiciones inversas: Cuba ha iniciado un proceso de apertura que la UE incentiva, mientras que Venezuela ha

* Doctora en Derecho Internacional Público y Master en Estudios Europeos por la Universidad Autónoma de Barcelona (UAB). Investigadora senior para América Latina de CIDOB desde 2002. Profesora asociada de Derecho Internacional Público en la UAB. Profesora asociada del Instituto Barcelona de Estudios Internacionales (IBEI). Miembro del Consejo Editorial de la Revista CIDOB d' Afers Internacionals; del Consejo Consultivo de la Revista Mural Internacional de la Universidade do Estado do Río de Janeiro; del Consejo Asesor del Comillas Journal for International Relations; y del Comité Científico de la revista América Latina Hoy. Es experta en América Latina.

** Profesora Contratada Doctora y Directora del Departamento de Ciencia Política y Relaciones Internacionales de la Universidad Autónoma de Madrid (UAM); Investigadora Asociada Senior de CIDOB, Barcelona. Susanne Gratius trabajó en diversos think-tanks en España (FRIDE, IRELA) y Alemania (GIGA, SWP) y fue profesora asociadada en el Departamento de Relaciones Internacionales de la Universidad Complutense de Madrid. Es experta en América Latina y la Unión Europea, imparte docencia en Máster y Grado sobre Análisis de Política Exterior, Teoría de Relaciones Internacionales y América Latina en el Sistema Internacional. Sus investigaciones y publicaciones se centran en América Latina (Cuba, Brasil y Venezuela) y sus relaciones con la UE (política exterior, regímenes políticos, populismo). 
Dossier América Latina: Venezuela transitado del populismo a un autoritarismo que Bruselas castiga. Otra razón serían las diferencias entre un Estado protector en Cuba y fragilidad estatal en Venezuela.

PALABRAS CLAVE: Cuba, Unión Europea, Venezuela, democracia, transiciones, sanciones.

\section{ABSTRACT}

The European Union (EU) has responded differently to the authoritarian regimes in Cuba and Venezuela - political dialogue and cooperation in the first case, selective sanctions and diplomatic break in the second. The article explores the reasons behind these different policies that contrast with the same goal - the promotion of democracy and human rights. One possible explanation are reverse transitions - Cuba started an opening process that the EU supports, whereas Venezuela initiated a path from populism to authoritarianism, sanctioned by Brussels. An additional reason could be the difference between a protective state in Cuba and state fragility in Venezuela.

KEYWORDS: Cuba, European Union, Venezuela, democracy, transitions, sanctions.

\section{Introducción}

En las últimas décadas, la Unión Europea (UE) ha desarrollado una política divergente hacia Cuba y Venezuela que señala inconsistencias en la defensa de los derechos humanos. Sin embargo, existen importantes diferencias entre los procesos políticos en ambos países que proporcionan argumentos para respaldar esa estrategia de la UE. Aunque Cuba y Venezuela están calificados como "países no libres" por Freedom House (2019) y el Índice Democrático del EIU (2019), los puntos de partida no son los mismos: en los últimos treinta años, Cuba inició un gradual y lento camino hacia una relajación del control económico y político, no para entrar en una transición democrática, sino para reinsertarse al mundo capitalista y adaptarse al creciente pluralismo político y económico. Venezuela recorrió el camino inverso: el chavismo llegó al poder por la vía electoral, transformó la democracia liberal en un régimen populista (Gratius, Rivero, 2018) y transitó durante la presidencia de Nicolás Maduro hacia un autoritarismo caótico (Ayuso, Gratius, 2016) con graves consecuencias socioeconómicas.

La estabilidad y el continuismo caracterizan al sistema político cubano que adoptó en 2019 una nueva constitución para consagrar la era poscastrista liderada por el presidente Miguel Díaz-Canel y el primer ministro Manuel Marrerero. Por el contrario, en Venezuela, el enfrentamiento entre oposición y oficialismo ha conducido a una estructura dual de poder que culminó en la "autoproclamación" de Juan Guaidó como presidente encargado del país en enero de 2019. Esta polarización interna se refleja también en la comunidad internacional que se divide entre un bloque liderado por los EE. UU. que apoyan a Guaidó y otro, encabezado por Cuba y Rusia, que sigue leal a Nicolás Maduro (Gratius, Puente, 2019). El claro 
apoyo del Parlamento Europeo (PE) y de la mayoría de los Estados miembros de la UE a Juan Guaidó y las sanciones selectivas que aprobó el Consejo contra miembros del gobierno de Maduro contrastan con el compromiso constructivo de su política de diálogo y cooperación con el régimen cubano con el cual firmó en 2016 un Acuerdo de Diálogo Político y Cooperación (ADPC) (Ayuso, Gratius, 2017). De este modo, a diferencia de Washington, la UE implícitamente no considera Cuba y Venezuela como regímenes similares: en el primer caso adopta una política propia y rechaza cada año ante las Naciones Unidas el embargo norteamericano a la Isla, y en el segundo sigue, con algunos matices, la estrategia de coerción de los EE. UU. (Ayuso, Gratius, 2020).

¿Cómo se pueden explicar estas respuestas diferentes de la UE a regímenes que obtienen la misma calificación de "autoritarios" (EIU, 2019) o "no libres" (Freedom House, 2019) en los índices democráticos? ¿Qué razones están detrás de estas políticas dispares? La hipótesis de que la relación triangular con los EE. UU. sea responsable de estas políticas se puede descartar, ya que en el mismo marco temporal la UE suscribe con matices el enfoque de Washington respecto a Venezuela, pero se distancia al dar una respuesta diferente en el caso cubano. Otra explicación sería el peso de España en la política latinoamericana de la UE en el sentido del uploading (Börzel, 2002), de europeizar su propia estrategia hacia determinados países de la región. Entre 1996-2016, España condicionó la política de la UE hacia Cuba mediante la Posición Común que suponía una doble condicionalidad democrática e impidió, durante estos veinte años, la firma de un acuerdo entre Bruselas y La Habana. En el caso venezolano, la relación plena de la UE durante la presidencia de Chávez (1999-2013) señaló igualmente una impronta española, en el sentido del compromiso constructivo, pero esta política se modificó en 2017 cuando, ante el creciente autoritarismo de Nicolás Maduro, España sugirió que la UE adoptara sanciones selectivas.

Una segunda hipótesis es que las diferentes respuestas de la UE responden a: a) una lógica de transiciones políticas inversas -apertura en el caso cubano y cierre autoritario en el venezolano; b) la estabilidad y continuidad que consolidan un Estado protector en Cuba, y el alto nivel de conflictividad que conduce a un Estado frágil en Venezuela. Para comprobar si estas explicaciones son ciertas, compararemos las políticas de la UE hacia ambos países entre 1999 y 2020 desde un enfoque teórico de transitología (cambio de un régimen a otro) y la promoción de la democracia. En términos metodológicos realizaremos, desde una perspectiva comparada de dos casos (Cuba y Venezuela), un análisis cualitativo de contexto basado en fuentes primarias (documentos oficiales, discursos, tratados) y secundarias.

\section{El debate sobre transiciones y promoción de la democracia}

Este artículo combina dos enfoques teóricos para explicar la política de promoción de la democracia de la UE con instrumentos diferentes hacia Cuba y Venezuela. En primer lugar, se basa en la literatura sobre transiciones democráticas y, más
Las respuestas de la Unión Europea a las transiciones inversas en Cuba y Venezuela

Anna Ayuso

Susanne Gratius 
Dossier América Latina: Venezuela

recientemente, del proceso inverso desde la democracia hacia el autoritarismo, teniendo en cuenta tanto los estudios clásicos de los años ochenta, donde encajaría el caso cubano, como a partir del debate actual sobre regímenes híbridos y retrocesos democráticos, donde se puede situar al régimen venezolano. Por otra parte, se tiene en cuenta la tesis de la paz democrática como explicación de la política de promoción de la democracia de la UE que comparte con EE. UU.

\section{Transiciones democráticas y autoritarias}

A partir de 2011, con el surgimiento de la llamada "Primavera Árabe", la investigación científica sobre transiciones hacia regímenes democráticos o autoritarios ha experimentado una importante recuperación de los trabajos previos realizados durante la tercera ola democrática en los años setenta del siglo XX. En estos cincuenta años ha surgido una amplia gama de estudios teóricos, por países y regiones, sobre las causas y consecuencias de la transición democrática (Morlino, 2015) -el complejo proceso del traspaso de un régimen político a otro-, las condiciones para consolidar democracias y las razones de su declive o caída, así como los instrumentos y objetivos de los actores internos y externos que influyen en este proceso. Abundan los estudios sobre: a) el tipo de regímenes (autoritarios, híbridos, democráticos); b) transiciones (liberalización, democratización y consolidación) en los casos de América Latina y el Sur de Europa; c) transformaciones (democracia y economía de mercado) en los países de Europa Central y Oriental.

De acuerdo con el creciente número de autocracias que entre 2000 y 2014 sustituyeron las democracias en 25 países (Diamond, 2015, p. 145), el debate más reciente se está centrando en los retrocesos democráticos o transición de la democracia liberal al autoritarismo (transiciones inversas) (Diamond, 2015; Erdmann, 2011; Levitsky, Ziblatt, 2018; Cassani, Tomini, 2019). El estudio de regímenes "híbridos" (Diamond, 2002), el proceso de "des-democratización" (Corrales, 2018) o de los “autoritarismos competitivos" (Levitsky, Way, 2010) ha sustituido las anteriores investigaciones comparativas sobre transiciones democráticas. Como afirman algunos autores, "la denominada tercera ola de democratización ha terminado y los académicos prestan cada vez más atención al aparente aumento del número de cambios de régimen en la dirección opuesta" (Cassani, Tomini, 2019, p. 121). ${ }^{1}$

Al haber sido clasificados como regímenes autoritarios y transitar en direcciones inversas, Cuba y Venezuela encajan en los tres debates sobre regímenes autoritarios, transiciones democráticas y retroceso autoritario. No obstante, Cuba ha sido una excepción en América Latina (Whitehead, 1996) que no figura en la literatura sobre la "Tercera Ola de Democratización" (Huntington, 1994) o los estudios sobre transición y democratización en los años ochenta y noventa (O’Donnell, Schmitter, Whitehead, 1986), ni tampoco aparece en el análisis sobre la promoción de la democracia de autores como Carothers, Magan, McFaul, Piccone o Youngs, entre otros.

\footnotetext{
1 Traducción propia.
} 
Por razones contrarias, Venezuela tampoco fue incluido en los estudios sobre transiciones y democracias en América Latina en los años ochenta y noventa, al haber sido una "excepción democrática" durante las dictaduras latinoamericanas que coincidió con el Pacto de Punto Fijo (1958-1999). Durante el periodo de Chávez (1999-2013), Venezuela es calificado de régimen híbrido en los índices de Freedom House y del EIU, aunque algunos estudios lo incluyen desde 2004 en la lista de retrocesos democráticos, calificándole de "autoritarismo competitivo" y "populismo autoritario" (Diamond, 2015, p. 145 y p. 151). Desde la presidencia de Maduro en 2013, Venezuela transitó definitivamente hacia el autoritarismo "caótico" (Ayuso, Gratius, 2016) con un Estado que no ofrece protección o servicios mínimos a los ciudadanos. Desde que Chávez asumió el poder en 1999, Venezuela ha sido incluido en los estudios sobre fragilidad estatal (Fund for Peace, 2019; International Crisis Group, 2019), populismo (Gratius, Rivero, 2018) y autoritarismo competitivo (Levitsky, Way, 2010).

\section{Promoción de la democracia}

La tercera ola democrática, aún durante la Guerra Fría, inició un amplio debate sobre la dimensión internacional de las transiciones democráticas (Whitehead, 1996) y un despliegue de numerosos instrumentos desde los principales promotores de la democracia, los EE. UU. y la UE, hacia las nuevas democracias, primero en el Sur de Europa, luego en América Latina y en la posguerra fría en los países ex comunistas de Europa Central y del Este. La Primavera Árabe permitió retomar y ampliar la dimensión externa de estos procesos mediante importantes estudios individuales y comparativos (entre otros, Youngs, McFaul, Carothers, Magen or Risse). No obstante, la tendencia global hacia el retroceso democrático, el auge de China y el declive del liderazgo de los EE. UU. han provocado que este campo de estudio se encuentre en "un estado de gran incertidumbre o incluso crisis” (Carothers, 2020, p. 121).

Junto a los Estados Unidos, la UE (sus instituciones y los Estados miembros) se ha perfilado como actor clave para promover la democracia y la paz en sus relaciones exteriores. Su política, igual que la de Washington, está fundamentada en la tesis liberal de la paz democrática, que se basa en la premisa de que las democracias no llevan a cabo guerras entre ellas y, por tanto, se justifica promover la democracia en terceros países como fórmula de paz (Doyle, 1997) extensible a la comunidad internacional. En los primeros veinte años de la post Guerra Fría, los EE. UU. y Europa invirtieron importantes recursos en programas de promoción de la democracia y los derechos humanos ${ }^{2}$ como instrumento de paz e integración en el orden internacional liberal. Aunque, al sopesar intereses y normas, la mayoría de los países percibió la promoción de la democracia como "un objetivo segundario de política exterior" (Spangler,

\footnotetext{
2 Como el European Instrument of Democracy and Human Rights (EIDH) entre 2014-2020.
}

Las respuestas de la Unión Europea a las transiciones inversas en Cuba y Venezuela

Anna Ayuso Susanne Gratius 
Dossier América Latina: Venezuela
Wolff, 2017, p. 5), ésta involucró a una gran variedad de actores e instrumentos, mecanismos de implementación y evaluación, sobre todo por parte de la UE y los EE. UU. (Carothers, 2020) que recuren a incentivos o sanciones.

Estas políticas contribuyeron a invertir la tendencia en 1988, cuando sólo un $35 \%$ de países (entre ellos Venezuela) fueron considerados democracias. Veinte años después, más del $45 \%$ de países eran clasificados como "libres" y sólo un 26 \% como autocracias (entre ellos Cuba) (Freedom House, 2019). Desde 2008, la crisis financiera y una redistribución de poder hacia potencias no democráticas, como China y Rusia, restó importancia y recursos a los programas de promoción democrática (Carothers, 2020). El auge de autocracias y regímenes híbridos, que juntos representan casi el 60 \% (Freedom House, 2019), se asocia con la influencia de China y Rusia y la crisis de la democracia en muchos países. Cuba y Venezuela no serían una excepción, sino parte de una tendencia que contesta la política de promoción democrática de Occidente.

Como potencia normativa (Manner, 2010), la UE proyecta valores, normas y principios como la promoción de la democracia y de los derechos humanos que forman parte de su naturaleza ${ }^{3}$, lo que se refleja en sus tratados y en los objetivos de su política exterior. El artículo 21 (título V, Capítulo 1) del Tratado de la Unión Europea recoge que: "La acción de la Unión en la escena internacional se basará en los principios que han inspirado su creación, desarrollo y ampliación y que pretende fomentar en el resto del mundo: la democracia, el Estado de Derecho (...)”. Asimismo, entre sus objetivos destaca: "b) consolidar y respaldar la democracia, el Estado de Derecho, los derechos humanos y los principios del Derecho internacional; c) mantener la paz, prevenir los conflictos y fortalecer la seguridad internacional" (Unión Europea, 26 de octubre de 2012).

En cuanto a los instrumentos de promoción de la democracia, cabe distinguir entre incentivos y sanciones. Entre los primeros destacan la membresía en ciertas organizaciones, la firma de acuerdos, la cooperación al desarrollo o la ayuda económica que entrarían en la categoría de incentivos o medidas positivas. Las cláusulas democráticas, la observación electoral, las declaraciones, las críticas públicas y las posiciones comunes serían más bien “incentivos negativos", midiendo sus consecuencias si el gobierno en cuestión abandonara la vía democrática. También existe una amplia gama de instrumentos de coerción que sancionan el comportamiento no democrático de terceros países. La UE contempla las sanciones como un instrumento esencial de la Política Exterior y de Seguridad Común (PESC) dentro de un "planteamiento político integrado y global que comprende el diálogo político, acciones complementarias y el recurso a otros instrumentos disponibles" (Consejo Europeo, s.f.) y siempre bajo los principios y normas del derecho internacional.

3 Según el artículo 10 del Tratado de la Unión Europea, "el funcionamiento de la Unión se basa en la democracia representativa” (Unión Europea, 26 de octubre de 2012). 


\section{Transiciones inversas: apertura y estabilidad en Cuba, autoritarismo y fragilidad en Venezuela}

Por distintas razones, Cuba y Venezuela son casos particulares en la región: por el carácter autoritario de sus regímenes políticos, sus transiciones inversas hacia apertura y estabilidad en el primer caso y el autoritarismo y el conflicto en el segundo, el papel de los militares, las sanciones de los Estados Unidos y de la UE en diferentes momentos históricos y el carácter binacional de sus Revoluciones. Aunque el presidente de los Estados Unidos, Donald Trump, ha calificado ambos regímenes de "dictaduras comunistas", hay importantes diferencias de origen, de objetivos y trayectorias entre los procesos y resultados de la Revolución cubana y la Revolución bolivariana. En primer lugar, el régimen castrista nació en 1959 de un conflicto armado contra la dictadura de Fulgencio Batista apoyada por los EE. UU. y tiene su legitimidad de origen en este período histórico. En Venezuela, el ex teniente coronel Hugo Chávez ganó las elecciones presidenciales de 1998, seis años después de un fallido intento de golpe de Estado contra el gobierno de Carlos Andrés Pérez. A pesar de esta diferencia, ambos regímenes comparten la legitimidad de carismáticos líderes hoy fallecidos: Fidel Castro y Hugo Chávez.

Otra diferencia radica en el carácter socialista y populista de las respectivas Revoluciones. Desde 1999, cuando Chávez asumió el poder e inauguró una alianza binacional con Cuba, la Revolución bolivariana tiene más características populistas que revolucionarias, aunque comparte con su "contraparte" cubana el mito refundacional, que en el caso venezolano se plasmó en la Constitución de 1999 aprobada por consulta popular. La Revolución cubana tiene un carácter socialista que, desde la imposición del embargo de los EE. UU. contra el castrismo en 1962, imitó en gran parte el modelo de la URSS, que hasta su disolución en 1990 fue el principal aliado externo de Cuba. La nueva constitución cubana de 2019 reafirma la irrevocabilidad del sistema socialista y que el partido socialista es único. Venezuela intentó, mediante un fallido referéndum en 2007, introducir el denominado Socialismo del Siglo XXI en la Constitución y expropió algunas empresas, pero la industria petrolera ya se había nacionalizado hacía décadas con la creación de Petróleos de Venezuela, S.A. (PDVSA) en 1975 como empresa pública por el gobierno de Carlos Andrés Pérez. El artículo 2 de la Constitución de Venezuela vigente afirma que "Venezuela se constituye en un Estado democrático y social de Derecho y de Justicia”. El carácter socialista del régimen chavista quedó reflejado principalmente en el nombre del partido oficialista PSUV y está presente en la retórica oficial, pero la ausencia de un Estado funcional la desmiente.

Un tercer elemento serían las transiciones inversas: un proceso gradual y controlado hacia una mayor apertura democrática y de economía de mercado en Cuba; y una evolución consciente de la democracia liberal hacia el populismo chavista y luego hacia el autoritarismo competitivo (Levitsky y Wahy, 2010) o caótico en Venezuela.

Las reformas que poco a poco erosionaron el monopolio político y económico del régimen castrista en Cuba empezaron en la época de Fidel que declaró
Las respuestas de la Unión Europea a las transiciones inversas en Cuba y Venezuela

Anna Ayuso

Susanne Gratius 
Dossier América Latina: Venezuela

el Período Especial en Tiempos de Paz y una Rectificación que consistió, entre 1990-1996, en medidas como el cuentapropismo, la dolarización de la economía, una reestructuración del sector agrícola o un mayor espacio para la Iglesia católica. En la segunda fase, ya durante el gobierno de Raúl Castro (2006-2018), se implementaron más reformas destinadas a permitir diferentes formas de propiedad, mayores libertades individuales de movimiento y pensamiento, un proceso de deliberación sobre el futuro de la Isla, la liberación de presos políticos y una descentralización del poder. Todos estos cambios se plasmaron en la Constitución de 2019, la cual tiene ciertas contradicciones ya que, por un lado, fomenta una mayor participación de los poderes locales y, por el otro, fusiona el liderazgo de los poderes ejecutivos y legislativos en manos del presidente que desde 2018 es Miguel Díaz-Canel. Otra novedad fue la creación de la función del primer ministro, posición que ocupa desde diciembre de 2019 Manuel Marrerero.

Todos estos cambios no han alterado la base de poder centralizado en torno al Consejo de Estado, el Consejo de Ministros y el unipartidismo, con una débil posición del Parlamento. La marcha atrás del proceso de liberalización de 1991-1996 con un retorno al autoritarismo mostró que la secuencia hacia la transición democrática no es lineal, y la posibilidad de un retroceso sigue existiendo. No obstante, desde la presidencia de Raúl Castro (2006-2018) hasta la de Miguel Díaz-Canel (2018-) ha habido un proceso de apertura política y económica gradual, pero constante en un marco de relativa estabilidad con un Estado consolidado que garantiza los servicios básicos, entre ellos seguridad, salud y educación, a los ciudadanos (Alonso, Vidal, 2019). Aunque en Cuba no se ha iniciado una transición democrática con pluralismo político y elecciones competitivas, como se muestra en la tabla 1, el proceso de cambios políticos desde 1990 ha conducido a un nuevo ciclo a medio camino entre el autoritarismo aún vigente y una incipiente y controlada liberalización.

Venezuela inició un camino político y económico muy diferente. Partió de una democracia liberal con mecanismos de participación democrática, aunque viciados de prácticas clientelares, y se convirtió en un régimen populista con un líder carismático que comunicaba directamente con el pueblo a través de programas televisivos como Aló Presidente. Chávez debilitó los balances institucionales de poder ganando poco a poco control sobre la empresa nacional PDVSA, los medios de comunicación, la justicia y los poderes electorales y legislativos (Gratius, Rivero, 2018). En esta transición a la inversa hacia una democracia "iliberal" (Zakaria, 1997) o un régimen híbrido (Diamond, 2015) y, en cualquier caso, populista ${ }^{4}$, Chávez gozaba de mayorías parlamentarias que le permitían gobernar por decreto mediante recurrentes habilitaciones que le excusaban de pasar por trámite parlamentario la mayoría de sus deci-

4 Populismo se define aquí como "un proyecto político antiliberal que altera la agenda de gobierno y las estructuras del poder, para refundar la democracia en torno a la unidad líder-pueblo sin necesidad de contar con instituciones representativas ni separación de poderes" (Gratius, Rivero, 2018, p. 36). 
siones. Desde que Nicolás Maduro asumió la presidencia, se transitó hacia un autoritarismo competitivo, especialmente tras perder las elecciones parlamentarias de 2015 en las que la oposición consiguió una mayoría absoluta que fue neutralizada mediante un uso abusivo de los poderes del Tribunal Supremo que declaró en desacato al parlamento. El chavismo se negó a compartir el poder con la oposición y creó una Asamblea Nacional Constituyente a su medida, aunque de forma inconstitucional.

\begin{tabular}{|c|c|c|}
\hline Período & Autoritarismo (no competitivo) & Liberalización (reforma y contra-reforma) \\
\hline $1988-1991$ & $\begin{array}{l}\text { Fidelismo (autoritarismo } \\
\text { carismático), Rectificación y } \\
\text { Período Especial en Tiempos de } \\
\text { Paz (programa de ajuste tras el } \\
\text { fin del bloque soviético CAME) }\end{array}$ & $\begin{array}{l}\text { Contra-liberalización a través de la } \\
\text { Rectificación (contra-modelo a Perestroika } \\
\text { y Glasnost), Ley Torricelli (sanciones } \\
\text { contra Cuba) en EE. UU., inicio de } \\
\text { relaciones diplomáticas con la UE (CAME) }\end{array}$ \\
\hline $1991-1996$ & $\begin{array}{l}\text { Estructura vertical de } \\
\text { poder Fidel y Raúl Castro, } \\
\text { continuidad institucional: } \\
\text { Consejo de Estado, Consejo de } \\
\text { Ministros, Buró Político, ANPP } \\
\text { (parlamento), partido único } \\
\text { PCC, Rectificación }\end{array}$ & $\begin{array}{l}\text { Liberalización: reforma constitucional, } \\
\text { más derechos individuales, espacio para } \\
\text { la Iglesia, pluralidad de pensamiento, } \\
\text { vanguardia del Centro de Estudios sobre } \\
\text { América (CEA); apertura económica y } \\
\text { mayores espacios para la iniciativa privada; } \\
\text { la UE como principal socio comercial }\end{array}$ \\
\hline 1996-2006 & $\begin{array}{l}\text { Represión contra disidentes, fin } \\
\text { de pluralismo de pensamiento, } \\
\text { retorno al autoritarismo } \\
\text { carismático, "batalla de ideas" } \\
\text { y alianza estratégica Cuba- } \\
\text { Venezuela }\end{array}$ & $\begin{array}{l}\text { Retroceso en reformas económicas, Ley } \\
\text { Helms Burton y Posición Común de la } \\
\text { UE (condicionalidad), política exterior } \\
\text { centrada en Venezuela, Raúl Castro } \\
\text { asume la Presidencia en } 2006\end{array}$ \\
\hline $2008-2020$ & $\begin{array}{l}\text { Represión selectiva y temporal } \\
\text { (intimidación), limitación de } \\
\text { actividades y actos culturales }\end{array}$ & $\begin{array}{l}\text { Lineamientos (2011), descentralización, } \\
\text { libertad de viajar, menos presos políticos, } \\
\text { ley de inversiones, más cuentapropistas } \\
\text { y PYMEs, modernización del sistema } \\
\text { productivo y empresarial, declive de } \\
\text { la alianza con Venezuela, relaciones } \\
\text { diplomáticas con EE. UU., ADPC con la } \\
\text { UE, cambio de Raúl a Miguel Díaz Canel } \\
\text { en } 2018 \text {, Constitución de } 2019\end{array}$ \\
\hline
\end{tabular}

Fuente: Elaboración propia.

Tras el proceso de reelección de Maduro en unos comicios presidenciales sin ningún tipo de garantías y una abstención histórica, se consolidó el régimen autoritario. La autoproclamación de Juan Guaidó como presidente encargado en respuesta a la elección fraudulenta de Maduro recibió el apoyo de gran parte de la comunidad internacional (EE. UU., Grupo de Lima, Unión Europea, entre otros) que reconoció al presidente de la Asamblea Nacional como legítimo presidente del país encargado de pilotar una transición a la
Las respuestas de la Unión Europea a las transiciones inversas en Cuba y Venezuela

Anna Ayuso

Susanne Gratius

Tabla 1.

Cuba entre autoritarismo y liberalización (1988-2020) 
Dossier América Latina: Venezuela
Tabla 2. Venezuela entre populismo y autoritarismo competitivo

(1999-2020) democracia. Esta dualidad de poder en un país que tiene dos presidentes y tres parlamentos (Gratius, 2019) no guarda relación con el partido único que sigue gobernando en Cuba con una cúpula política hermética y una oposición débil o en el exterior.

Un cuarto elemento que marca distancias es el peso del Estado como factor de estabilidad (Cuba) e inestabilidad (Venezuela). Según el Índice de Estados Frágiles de 2019, Cuba ocupa la posición 118 y es considerado, junto a Grecia y Chipre, como "más estable" y mejor situada que, por ejemplo, la República Dominicana, Perú o México (Fund for Peace, 2019). Venezuela se sitúa 86 posiciones atrás, ocupando el $32^{\circ}$ lugar calificado de "advertencia alta" en la frontera de "alerta", categoría que comparte con Guatemala. Desde el inicio, Cuba creó un Estado protector que garantiza la seguridad

\begin{tabular}{|c|c|c|}
\hline Período & Populismo & Autoritarismo competitivo o caótico ${ }^{\mathrm{a}}$ \\
\hline 1999-2006 & $\begin{array}{l}\text { Liderazgo carismático de Hugo } \\
\text { Chávez, acto refundacional: } \\
\text { Constitución } 1999 \text { que introduce } \\
5 \text { poderes (ejecutivo, legislativo, } \\
\text { judicial, moral, electoral), "Aló } \\
\text { Presidente": diálogo presidente- } \\
\text { pueblo, "soberanía popular" } \\
\text { y "democracia participativa", } \\
\text { creación de Misiones y poderes } \\
\text { locales, elecciones, acuerdos con } \\
\text { Cuba, avances sociales }\end{array}$ & $\begin{array}{l}\text { Mayor represión y polarización } \\
\text { después del intento de golpe de Estado } \\
\text { 2002, restricciones a leyes y normas } \\
\text { electorales (nuevas circunscripciones), } \\
\text { inhabilitación e intimidación de } \\
\text { opositores, concentración de poder en } \\
\text { manos del presidente y debilitamiento } \\
\text { de Estado de Derecho y controles } \\
\text { institucionales (poderes ciudadano, } \\
\text { electoral, judicial y legislativo ocupados } \\
\text { por leales al Gobierno) }\end{array}$ \\
\hline 2007-2013 & $\begin{array}{l}\text { Elecciones y consultas populares, } \\
\text { reforma Constitucional } \\
2007 \text { rechazada, paulatino } \\
\text { debilitamiento de populismo, } \\
\text { enfermedad de Hugo Chávez, } \\
\text { mayor participación de Cuba } \\
\text { en la Revolución Bolivariana, } \\
\text { Misiones }\end{array}$ & $\begin{array}{l}\text { Nacionalizaciones de empresas, control } \\
\text { de precios, decretos presidenciales } \\
\text { para introducir "el Socialismo del Siglo } \\
\text { XXI" en Venezuela, persecución y } \\
\text { encarcelamiento de opositores (presos } \\
\text { políticos), sanciones selectivas EE. UU. }\end{array}$ \\
\hline 2013-2020 & $\begin{array}{l}\text { Post-populismo: legitimidad } \\
\text { de Nicolás Maduro como } \\
\text { sucesor o "heredero" de Chávez } \\
\text { (designación en La Habana), } \\
\text { encarnación del chavismo y del } \\
\text { líder carismático (2013-2015), } \\
\text { el "hijo de Chávez, movimiento } \\
\text { chavista y fortalecimiento del } \\
\text { partido PSUV }\end{array}$ & $\begin{array}{l}\text { Lucha de poder entre madurismo y } \\
\text { oposición, empate desde } 2015 \text {, cuando } \\
\text { la oposición gana la mayoría en la AN, } \\
\text { intento de cerrar la AN, disidencia } \\
\text { dentro de chavismo, elecciones } \\
\text { fraudulentas o sin oposición, dualidad } \\
\text { de poder ejecutivo (Maduro/Guaidó) } \\
\text { y legislativo (AN dividida, ANC), } \\
\text { colapso económico, crisis humanitaria, } \\
\text { sanciones EE. UU. (embargo) y UE }\end{array}$ \\
\hline
\end{tabular}

${ }^{\text {a }}$ Ayuso, Gratius, 2016.

Fuente: Elaboración propia. 
ciudadana y la protección social. El resultado son sistemas universales públicos de salud y educación, la "cartilla" que ofrece una canasta de alimentos básicos (aunque se ha ido reduciendo) y un sistema de seguridad social con pensiones mínimas y protección ante el desempleo. Este Estado protector no existe en Venezuela donde los servicios sociales son muy precarios y fueron sustituidos por las "misiones", programas sociales focalizados creados con la ayuda de Cuba y la Fuerza Armada Nacional Bolivariana (FANB) y los Comités Locales de Abastecimiento y Producción (CLAP) que son ayudas alimentarias clientelares y arbitrarias.

Venezuela ha sido calificado de "Estado frágil" por el Fund for Peace e International Crisis Management. El informe de la Alta Comisionada de las Naciones Unidas para los Derechos Humanos, Michelle Bachelet, denunció una "severa crisis humanitaria" (ACNUDH, 2019). En función de esta diferencia trascendental, Cuba representa estabilidad en la región e incluso ha actuado de mediadora para facilitar la paz en Colombia con las negociaciones entre el gobierno y FARC -afines a la Revolución cubana- que tuvieron lugar en La Habana. Esta estabilidad contrasta con el conflicto venezolano que ha dividido a los países del continente americano entre una minoría (Argentina, Cuba, Nicaragua o México) que reconocen a Nicolás Maduro como presidente del país y una mayoría, integrada en el Grupo de Lima, que considera al opositor Juan Guaidó como máxima autoridad del país.

A pesar de coincidencias ideológicas por ser regímenes que se definen por su oposición al imperialismo capitalista, por su legitimidad de origen en un líder carismático y el peso de las Fuerzas Armadas, subsisten diferencias entre ambos procesos autodenominados como revolucionarios. Comparten debilidades como la dependencia de la exportación de un recurso estratégico -Cuba de la exportación de recursos humanos y Venezuela de petróleo- y de las remesas y ambos enfrentan sanciones de los Estados Unidos y comparten la alianza con China y Rusia y la membresía de la Alianza Bolivariana para los Pueblos de Nuestra América (ALBA). Eso los convierte en aliados, pero no en iguales. Para la UE el objetivo de fomentar un proceso democrático y los derechos humanos es el mismo en ambos países, pero se aplican instrumentos diversos. ¿Significa eso que el criterio aplicado por la UE no depende de los resultados, sino que se evalúa la dirección que toma el proceso? Un factor determinante de la posición europea vendría dado por el nivel de conflictividad, en el sentido de aplicar un compromiso constructivo si el nivel de conflicto es bajo y hay mejoras, y se recurre a la coerción y la presión diplomática cuando hay un alto nivel de conflictividad, como en el caso venezolano. En este ensayo nos proponemos hacer una lectura explicativa de la lógica detrás de las políticas de la UE hacia Cuba y Venezuela. Partimos de un enfoque racional para explicarlas, aunque no se pueden obviar factores emocionales como, por ejemplo, la relación especial que mantiene España con ambos países.
Las respuestas de la Unión Europea a las transiciones inversas en Cuba y Venezuela

Anna Ayuso

Susanne Gratius 


\section{Dossier \\ América Latina: \\ Venezuela \\ Las políticas de la UE hacia Cuba y Venezuela: compromiso versus coerción}

La UE ha ido adaptando sus políticas hacia Cuba y Venezuela en función de los cambios políticos que se han producido en ambos países, pero también a la presión e influencia de diversos actores. En ambos casos, la UE modificó las políticas aplicadas según los objetivos y aplicó una combinación de acciones y decisiones que dependen de la constelación de actores y la coyuntura interna y externa. Esta última incluye necesariamente la influencia de las políticas de los EE. UU. hacia ambos países y la respuesta del resto de los países de la región. Por ejemplo, la UE siempre se ha mantenido alineada con la mayoría de los países de América Latina en el rechazo a los efectos extraterritoriales de la Ley Helms-Burton, en cambio se ha alineado con los Estados Unidos y el Grupo de Lima en el reconocimiento de Guaidó. Por tanto, se pueden identificar elementos comunes y diferenciales, tanto en cuanto a los objetivos, como en lo referente a los instrumentos en las orientaciones políticas aprobadas por el Consejo de la Unión Europea en combinación con el Parlamento Europeo y la Comisión.

\section{Las diferentes "voces” en la Unión Europea}

Al no ser un actor cohesionado, la UE representa voces divergentes frente a terceros. Asimismo, hay una clara división de competencias entre el Parlamento Europeo (PE) que vela sobre la democracia y los derechos humanos, el Consejo y los Estados miembros que determinan posiciones comunes e imponen sanciones, la Comisión Europea que ejecuta las políticas acordadas y las representa a través del alto representante y vicepresidente en el exterior. No obstante, tanto en la política de la UE hacia Cuba como hacia Venezuela ha habido inconsistencias, paradojas y posiciones divergentes que han obstaculizado un papel más claro o destacado de la UE y/o revelaron un disenso interno.

En ambos países, el Consejo de la Unión Europea que representa los Estados miembros, es la institución determinante para adoptar medidas hacia los gobiernos en Cuba y Venezuela. En el caso de Cuba, adoptó dos instrumentos: entre 1996 y 2016 la Posición Común de la UE que introdujo una "doble condicionalidad democrática" (Gratius, 2017) y en 2014 aprobó el mandato de negociación de un acuerdo de cooperación con Cuba. En el caso de Venezuela propuso el Grupo Internacional de Contacto para facilitar una transición negociada, y decidió desde 2017 implementar sanciones selectivas contra miembros del gobierno de Maduro. Desde la aprobación de la Posición Común de 1996, que se había aprobado a iniciativa de España, hasta 2014 el Consejo debatió el caso cubano cada año para reevaluar la Posición Común. Finalmente, en 2014, el Consejo autorizó el mandato de negociación de la Comisión Europea para firmar, en 2016, un acuerdo de cooperación con Cuba. 
En el caso de Venezuela, el Consejo emitió siete Declaraciones, todas ellas incluyendo un llamado al diálogo, a una solución pacífica del conflicto y a una salida electoral. El 18 de julio de 2016, el Consejo apoyó la mediación de tres expresidentes, D. José Luis Rodríguez Zapatero, D. Leonel Fernández y D. Martín para "facilitar un diálogo urgente, constructivo y efectivo entre el Gobierno y la mayoría parlamentaria en Venezuela”. Un año después, el Consejo adoptó la Decisión (PESC) 2017/2074 de 13 de noviembre de 2017, con "profunda preocupación por el continuo deterioro de la democracia, el Estado de Derecho y los derechos humanos y el ataque a la Asamblea Nacional" y decidió imponer medidas restrictivas selectivas contra primero siete individuos y, desde 2019, ya 25 miembros del gobierno venezolano. Paralelamente a las sanciones, el Consejo de la Unión Europea lanzó el Grupo Internacional de Contacto (GIC) (Consejo de la Unión Europea, 31 de enero de 2019) sobre Venezuela que se reúne desde el 7 de febrero de 2019, con el fin de apoyar la mediación que asumió Noruega. Ocho países europeos y cuatro latinoamericanos ${ }^{5}$ participan en el GIC para buscar una salida negociada en un plazo previsible y proponer una serie de medidas de fomento de la confianza ${ }^{6}$ que concluyan con una transición política y elecciones presidenciales limpias. Hasta ahora, sus resultados han sido limitados.

En ambos casos, existían importantes divisiones entre los Estados miembros que representan el Consejo, y España fue un actor clave para adoptar las respectivas políticas del Consejo. Así, tanto la Posición Común de la UE sobre Cuba como la adopción de restricciones en 2003, su levantamiento en 2008 y el mandato de negociación de un acuerdo en 2014 fueron iniciativas de España, cuyos cambiantes gobiernos (PP o PSOE) alteraron el enfoque hacia Cuba y Venezuela, hacia una posición más crítica o cooperativa. Por otra parte, el reconocimiento de Juan Guaidó como presidente de Venezuela dividió a la UE (Ayuso, Gratius, 2019) y, en febrero de 2019, seis Estados miembros (Italia, Grecia, Irlanda, Rumanía, Eslovaquia y Chipre) no siguieron la política del grupo en torno a España que de facto rompió con el gobierno de Maduro. En el caso cubano, la oposición de países como España durante el gobierno de Aznar (1996-2004), de Alemania y de la República Checa impidió durante veinte años eliminar la Posición Común de la UE sobre Cuba (Ayuso, Gratius, 2017).

El Parlamento Europeo, al poner el acento en la democracia y los derechos humanos, concedió el Premio Sájarov a la oposición en Cuba $(2005,2010)$ y Venezuela (2017). Antes de 2013, el PE dedicó una atención similar a la

5 Bolivia, Costa Rica, Ecuador, Panamá, Alemania, España, Francia, Italia, Países Bajos, Portugal, Suecia y el Reino Unido.

6 Tales como: la liberación de los presos políticos y el levantamiento de la prohibición a los políticos opositores presentarse como candidatos; respeto del papel constitucional de la Asamblea Nacional; ayuda exterior para responder a las necesidades de la población; una composición equilibrada del Consejo Nacional Electoral; estímulo y una ayuda a la oposición para que supere sus divisiones internas y coopere.
Las respuestas de la Unión Europea a las transiciones inversas en Cuba y Venezuela

Anna Ayuso

Susanne Gratius 
Dossier América Latina: Venezuela

Tabla 3.

Número de Resoluciones del PE sobre la situación política en Cuba y Venezuela

situación de derechos humanos en Cuba y en Venezuela, y después siguió políticas diferentes hacia ambos regímenes. En el caso de Cuba, el PE había sido un actor clave para iniciar una mayor presión democrática sobre el régimen y votó a favor de la Posición Común de la UE sobre Cuba, aprobada en diciembre de 1996. A partir de entonces, hasta 2014, todas las resoluciones del PE sobre Cuba señalan la prioridad de los derechos humanos y del apoyo a la oposición. Este enfoque cambió en 2014, paralelo a la aprobación del mandato de negociación de un acuerdo UE-Cuba por parte del Consejo. Durante los años siguientes, el PE acompañó a la Comisión Europea en el proceso de negociación con Cuba (Ayuso, Gratius, 2017) sin desviarse de la posición oficial de concluir el acuerdo bilateral que el PE aprobó, sin abrir un debate sobre derechos humanos y oposición. La Resolución legislativa del 5 de julio de 2017 constata que "la estructura, el contenido y la dinámica del Acuerdo se adaptan a los principios y valores establecidos por las instituciones de la Unión”. Asimismo, afirma "el valor estratégico de la relación entre la Unión y Cuba". De esta manera quedó justificado por parte del PE seguir una política de compromiso constructivo con el régimen cubano, lo cual contrastó con anteriores posiciones de apoyo e interlocución de los opositores.

En cuanto a Venezuela, a partir de 2013, el PE inició una política constante de denuncia contra el gobierno de Maduro mediante doce resoluciones sobre Venezuela, todas ellas críticas con la situación de derechos humanos y destacando el declive de seguridad humana. En el período 1999-2020, el PE aprobó 20 resoluciones sobre Venezuela, la mayoría en el año 2019, como respuesta al conflicto entre "los dos presidentes". El PE fue la primera institución de la UE que reconoció, sin condiciones previas, en su resolución del 31 de enero, a Juan Guaidó como "legítimo presidente" del país, antes de iniciarse el debate sobre este asunto entre los Estados miembros de la UE.

La Comisión Europea asumió un importante papel en materia de cooperación al desarrollo y ejecutora de las políticas definidas por el Consejo y el PE. A pesar de estar catalogado como un país de renta media alta por la Organización de Cooperación para el Desarrollo Económico (OCDE), Cuba se beneficia de cooperación bilateral mediante el Programa Indicativo Multianual

\begin{tabular}{lcc}
\hline Período Legislativo & Cuba & Venezuela \\
\hline $\begin{array}{l}\text { 2019-2024 (hasta mayo } \\
2020)\end{array}$ & 1 & 2 \\
\hline $2019-2014$ & 3 & 9 \\
\hline $2009-2014$ & 1 & 4 \\
\hline $2004-2009$ & 3 & 3 \\
\hline $1999-2004$ & 3 & 2 \\
\hline Total & 11 & 20 \\
\hline
\end{tabular}

Fuente: Búsqueda: 1999-2020: sistema de búsqueda Resoluciones del PE en: https://www.europarl. europa.eu/plenary/es/texts-adopted.html\#sidesForm y selección de Resoluciones sobre la situación política. 
2014-2020 (Servicio Europeo de Acción Exterior, 2014) a través del cual la UE destinó un fondo total de 50 millones de euros. A estos se suman otras acciones procedentes de otras fuentes de financiamiento de la UE, incluyendo la cooperación regional. La presencia de Cuba en la cooperación multilateral de la Comisión Europea hacia la región es el efecto del Acuerdo de Diálogo Político y Cooperación (ADPC), ya que anteriormente Cuba no tuvo acceso a los programas regionales y entre 2003 y 2008 la cooperación europea se había cortado, debido a las medidas restrictivas que la UE introdujo en la política hacia Cuba tras una nueva ola de represión en la Isla.

Similar al estatus de Cuba, al ser calificado como país de renta media alta por la OCDE, Venezuela no es un destino importante de la cooperación europea. En 2018, la Comisión Europea sólo financió tres proyectos y un periodo de tres años sobre prevención y resolución de conflictos. Entre 2007 y 2013, la UE cofinanció pocos proyectos con sólo 40 millones de Euros (6,6 millones anuales). Estos se centraron en la modernización del Estado y la diversificación económica, con un pequeño aporte para la lucha contra la droga. Una vez se hizo evidente la crisis humanitaria, en agosto de 2018, la CE prometió un paquete de 35 millones de euros en ayuda humanitaria, adicional a los 37 millones ya previstos. Sin embargo, el régimen la rechazó, ya que la ayuda humanitaria ofrecida por los EE. UU., la UE y otros países que apoyan a Guaidó se convirtió en un foco del conflicto interno.

En su Declaración del 3 de abril de 2020, el alto representante Josep Borrell señaló por primera vez las coincidencias políticas entre los EE. UU. y la UE frente a la crisis venezolana al destacar que "la Unión Europea acoge favorablemente el Marco de Transición Democrática de Venezuela propuesto por los Estados Unidos. Esta propuesta está en la misma línea que la de la UE, que aboga por una solución pacífica a la crisis por medio de una negociación que dé lugar a un gobierno democrático, que resulta más necesario que nunca en este momento". El marco de transición para Venezuela que presentó el gobierno de los EE. UU. el 31 de marzo de 2020 prescribe los diferentes pasos de la transición bajo la tutela de la Asamblea Nacional presidida por Juan Guaidó y el gradual levantamiento de las sanciones de los EE. UU. y de la UE (US Department of State, 31 de marzo de 2020). Este apoyo público de la UE al plan de transición diseñado por el gobierno de Trump contrasta con el claro rechazo de un plan similar presentado desde 2005 por el Coordinador de la Transición en Cuba, Caleb McCarry, durante el gobierno de George W. Bush para cambiar el régimen castrista, lo cual indica un cambio de políticas.

Analizando la evolución de las posiciones de las instituciones de la UE hacia Cuba y Venezuela se observa una evolución inversa hacia ambos países que ha llevado a fortalecer la cooperación con Cuba y a adoptar medidas coercitivas selectivas con Venezuela. Asimismo, se observa un distanciamiento de políticas entre los EE. UU. y la UE en el caso cubano y una convergencia de posiciones en el caso de Venezuela (Ayuso, Gratius, 2020). Si bien el objetivo
Las respuestas de la Unión Europea a las transiciones inversas en Cuba y Venezuela

Anna Ayuso

Susanne Gratius 
Dossier América Latina:

Venezuela

declarado respecto a ambos países es el mismo, la protección de los derechos humanos y el restablecimiento de la democracia, las estrategias y los instrumentos no son iguales. La UE ha ido adaptando instrumentos de presión e incentivos en función de las circunstancias y la evolución de los acontecimientos hacia una política de condicionamiento, compromiso y coerción.

\section{Condicionamiento, compromiso y coerción}

En el caso de Cuba han predominado tres paradigmas externos para promover la democracia -todos ellos con resultados limitados-: un enfoque de "compromiso constructivo" por parte de América Latina, Canadá y, desde 2014, por la UE para promover un proceso gradual de reformas desde arriba; una política de sanciones unilaterales y de cambio de régimen desde los EE. UU.; y el "compromiso condicionado" de la Posición Común de la UE entre 1996 y 2016 (Gratius, 2005, 2017), que condicionaba la cooperación a avances en la democratización. El elemento diferenciador es la combinación entre elementos coercitivos e incentivos con instrumentos jurídicos, políticos y de cooperación.

En el caso de Venezuela, la UE se mantuvo en el plano diplomático y de condicionalidad de la ayuda hasta que optó por una política de coerción, al imponer desde 2017 sanciones selectivas y un embargo de armas que sustituyó a la anterior estrategia de compromiso constructivo. Sin embargo, aun recurriendo a las sanciones, la UE ha mantenido una agenda política de búsqueda de alianzas que faciliten una salida democrática al conflicto entre el chavismo y la oposición. Eso se ha materializado en el liderazgo del GIC que busca apoyar una solución negociada. Las primeras sanciones no se aplicaron hasta noviembre de 2017, pero previamente ya se habían ido aprobando resoluciones por parte del Consejo, expresando preocupación por el deterioro de la situación a raíz de las elecciones presidenciales de 2013 y la represión que siguió a las protestas de la oposición.

\section{Compromiso constructivo}

La estrategia que hoy sigue la UE con Cuba es la del compromiso constructivo, entendido como una cooperación sin condiciones previas. Cuando, en febrero de 2014, el Consejo dio luz verde a la negociación de un acuerdo de cooperación con Cuba (Consejo de la Unión Europea, 10 de febrero de 2014), no se derogó la posición común. De hecho, en el mandato se recogían las palabras de la entonces alta representante Catherine Ashton que condicionaba la negociación a las reformas democráticas ${ }^{7}$. La derogación de la posición solo se hizo

\footnotetext{
7 "Esta decisión no constituye un cambio político respecto al pasado. Del mismo modo que queremos apoyar la reforma y la modernización en Cuba, hemos planteado de manera coherente
} 
cuando el Consejo dio su consentimiento a la firma de un ADPC con la Isla con la Decisión del Consejo (PESC) 2016/2233 (Consejo de la Unión Europea, 6 de diciembre de 2016). El acuerdo fue firmado el 12 de diciembre después de que hubiera sido aprobado en el Parlamento Europeo con 567 votos a favor, 61 en contra y 31 abstenciones. En esta ocasión, la entonces Alta Representante Federica Mogherini, con motivo de la entrada en vigor provisional del acuerdo, señaló que: "Hoy la Unión Europea se acerca a Cuba y a todos los ciudadanos cubanos, cuando el país experimenta una modernización económica, política y social" (Comisión Europea, 31 de octubre de 2017).

Desde la entrada en vigor se han realizado dos Consejos conjuntos, el último el 9 de septiembre de 2019 en La Habana donde se establecieron cinco diálogos políticos estructurados ${ }^{8}$. El objetivo es favorecer el proceso de transición democrática pilotado desde dentro, un aumento del respeto a los derechos y libertades de la oposición y una creciente apertura económica y social. Para este fin, el ADPC incluye un diálogo sobre derechos humanos, que es el punto más sensible de las relaciones (Ayuso, Gratius, 2017). Es pronto para valorar los resultados de esta nueva estrategia, pero por el momento se ha producido una transición a una nueva generación de dirigentes de forma gradual, aunque en condiciones económicas desfavorables debido a nuevas sanciones por parte de la administración Trump que sigue una política opuesta a la europea. Tras el anuncio de la activación del Título III de la Ley Helms-Burton -las sanciones extraterritoriales aprobadas en 1996 y suspendidas desde entonces- a partir de mayo de 2019 por parte del gobierno de los EE. UU., la UE se opuso en una carta firmada por Federica Mogherini, y la Comisaria Europea de Comercio, Cecilia Malmström, a la "aplicación extraterritorial de medidas restrictivas unilaterales contrarias al derecho internacional" y afirmaba que "considerará todas las opciones a su alcance para proteger sus intereses legítimos (...) a través del uso del 'estatuto de bloqueo' de la UE”. Esto confirma que la UE siempre se ha opuesto a las medidas coercitivas con efectos extraterritoriales, y sus Estados miembros votan cada año en las Naciones Unidas contra el embargo de los EE. UU.

En el caso de Venezuela, la UE adoptó una política de compromiso constructivo durante el gobierno de Hugo Chávez (1999-2013), cuando mantuvo relaciones económicas y diplomáticas plenas, sin ningún tipo de condicionamiento o medidas restrictivas. Esta estrategia se mantuvo durante los primeros años de Maduro, cuando la UE apoyó la mediación de los tres expresidentes, pero tras el fracaso de esta mediación y un nuevo intento del Vaticano, así como la convocatoria de la Asamblea Constitu-

nuestras preocupaciones en materia de derechos humanos que seguirán siendo centrales en esta relación. Espero también que Cuba haga lo necesario por su parte, especialmente en materia de derechos humanos. El ritmo de nuestras negociaciones será reflejo de esta actuación.”

8 En materia de: derechos humanos, no proliferación de armas de destrucción masiva, control de armas convencionales, medidas restrictivas unilaterales y desarrollo sostenible.
Las respuestas de la Unión Europea a las transiciones inversas en Cuba y Venezuela

Anna Ayuso

Susanne Gratius 
Dossier América Latina: Venezuela yente en 2017, la UE empezó a aplicar sanciones selectivas y pasó a una fase más coercitiva.

\section{Compromiso condicionado}

La Posición Común de 1996 establecía en el primer punto que: "El objetivo de la Unión Europea en sus relaciones con Cuba es favorecer un proceso de transición hacia una democracia pluralista y el respeto de los derechos humanos y libertades fundamentales, así como una recuperación y mejora sostenibles del nivel de vida del pueblo cubano." Y añadía "la política de la Unión Europea no contempla provocar el cambio mediante la aplicación de medidas coercitivas que tengan por efecto incrementar las dificultades económicas del pueblo cubano" (Consejo de la Unión Europea, 1996). Por tanto, se descartaba el cambio de régimen y se apostaba por la transición pacífica. Como instrumentos se establecía la intensificación del diálogo con gobierno y sociedad para fomentar los derechos humanos, la eliminación de los delitos políticos, la liberación de los presos políticos y el cese del hostigamiento a los disidentes y, como incentivo, se mostraba dispuesta a intensificar la cooperación económica "a medida que las autoridades cubanas avancen hacia la democracia”. Por ello, se estableció un proceso de revisión a los seis meses. Es decir, a pesar de que se recortó la cooperación con el gobierno, se mantuvo el diálogo político y la posibilidad de una cooperación según la evolución de los acontecimientos.

En contraste con la vigente estrategia, la posición común de la UE hacia Cuba en el periodo 1996-2016 se puede calificar de compromiso condicionado, ya que el objetivo era igualmente promover un proceso de transición democrática y el respeto a los derechos humanos, pero se condicionaba la cooperación a cambios efectivos en la democratización del régimen. Como instrumentos se mantuvo el diálogo político, pero la cooperación se limitó a la ayuda humanitaria y en el plano de diplomacia pública se mantuvo el apoyo a la oposición que se tradujo en un importante activismo del PE. Sin embargo, nunca se llagaron a aplicar sanciones a Cuba y eran muchos los Estados miembros que mantenía relaciones estables de diálogo y comercio. Esta política evolucionó, después de que iniciativas de la Iglesia católica, con el apoyo de España, contribuyeron a la liberación de presos políticos y sobre todo cuando a través del diálogo político se llegó al ADPC. Hubo un efecto de europeización de política hacia Cuba ya desde el periodo de Ashton, pero sobre todo con Mogherini. Tampoco se puede obviar la influencia del cambio de orientación de la Administración Obama hacia Cuba que facilitó el cambio de postura de países europeos más reacios al cambio de estrategia.

En el caso de Venezuela, la situación fue a la inversa. El fracaso de las iniciativas diplomáticas y la profundización del deterioro institucional con el ataque a los poderes de la Asamblea Nacional llevó a un endurecimiento de 
la postura de la UE ya abiertamente crítica con el régimen. Esto conllevó a la adopción de las sanciones selectivas en 2017 y se dio un periodo transitorio en el que se pusieron las esperanzas en la mediación de Noruega que acabó en un nuevo fracasó y llevó al endurecimiento de la posición europea que condujo a la ruptura de relaciones con Maduro tras el reconocimiento de Juan Guaidó como presidente encargado en enero de 2019.
Las respuestas de la Unión Europea a las transiciones inversas en Cuba y Venezuela

Anna Ayuso

Susanne Gratius

\section{Política de coerción}

Como se señaló, nunca se llegaron a aplicar sanciones por parte de la UE hacia Cuba e incluso se mantuvo una posición crítica con el embargo norteamericano, aunque hubo tensión política durante los periodos más duros de represión a la disidencia, particularmente en 2003. En cambio, en el caso de Venezuela la UE sí ha adoptado medidas unilaterales y se alineó con los Estados Unidos en el reconocimiento de Guaidó y, por tanto, la ruptura de las relaciones con el gobierno de Maduro. Sin embargo, Bruselas difiere de Washington en el tipo de sanciones, en el rechazo de una intervención militar y en el impulso de un diálogo internacional para facilitar una transición pacífica. Respecto a la actual situación de Venezuela, se está produciendo un cierto repliegue en el pulso de presión al tenerse en cuenta las consecuencias que las sanciones están teniendo en el agravamiento de las condiciones de vida de la población, especialmente tras el estallido de la pandemia del COVID 19. Esto ha hecho que se plantee incrementar la cooperación de tipo humanitaria, aunque de momento no se levantan las sanciones selectivas, pero tampoco se incrementan como sí ha hecho la Administración Trump.

\section{¿Son las transiciones inversas y el nivel de conflictividad los factores que explican las diferentes respuestas de la UE?}

Esta comparación de políticas permite llegar a las siguientes conclusiones. Primero, la política de la UE hacia Cuba y Venezuela coincide en los objetivos, pero no en la estrategia y los instrumentos que, desde 2017, son diametralmente opuestos: compromiso constructivo y cooperación con el régimen cubano y coerción, sanciones y ruptura de relaciones diplomáticas con el régimen venezolano. No obstante, como revela la tabla 4, la UE ha aplicado ambas políticas, compromiso y coerción, en diferentes momentos hacia Cuba y Venezuela, de modo que las diferencias sólo se perciben en los últimos tres años, desde la aprobación de sanciones selectivas por parte del Consejo.

En cuanto a los objetivos, la situación de conflictividad en Venezuela añade un elemento adicional, la solución pacífica, a la promoción de la democra- 


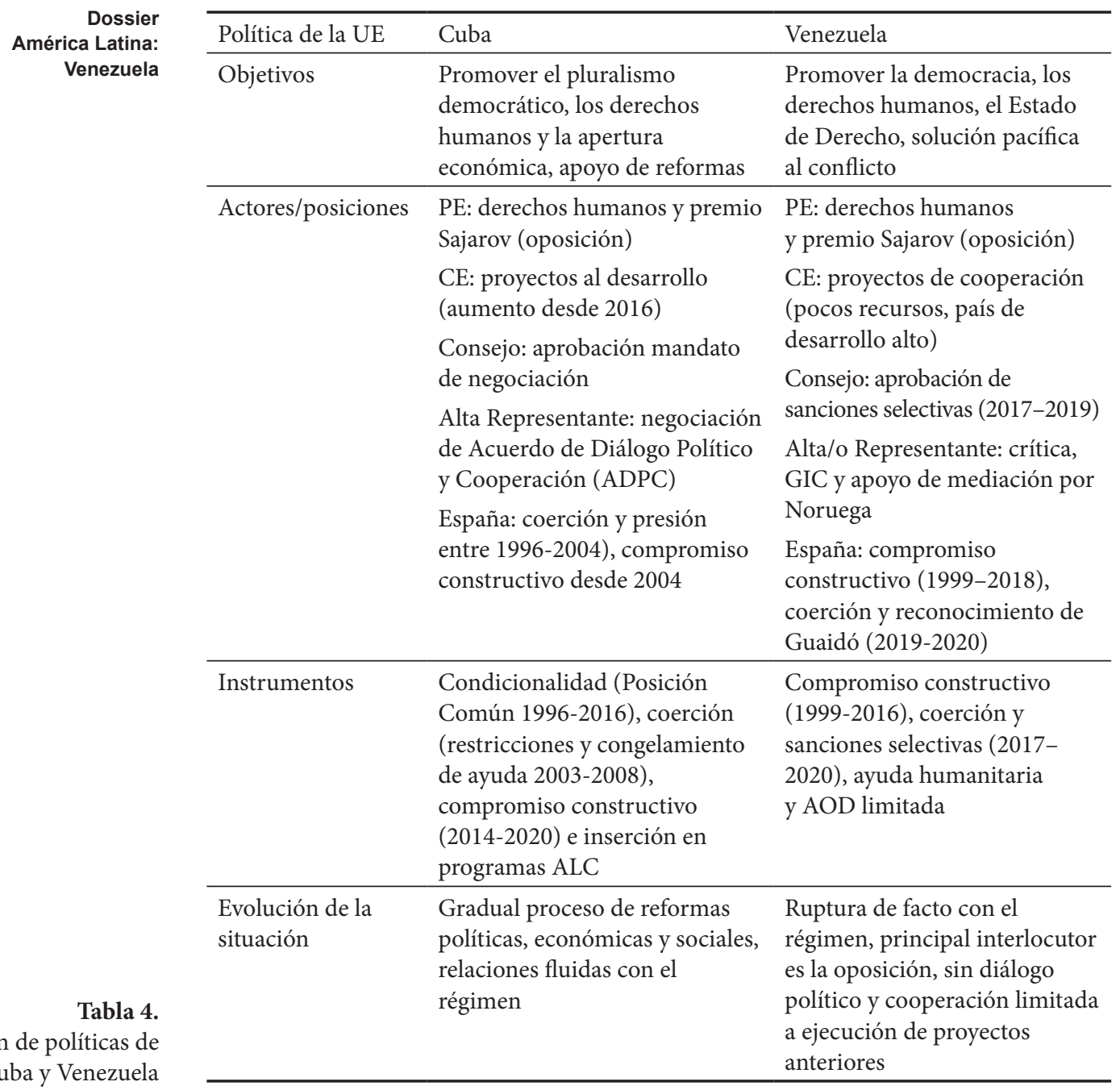

(2013-2020)

Fuente: elaboración propia.

cia y los derechos humanos que también han guiado, según el discurso oficial, la estrategia de la Unión hacia Cuba. Así, la UE ha centrado su política hacia Venezuela en el alto nivel de conflictividad y en la crisis humanitaria que en el caso venezolano indica una fragilidad estatal que contrasta con un Estado consolidado y una mayor estabilidad en Cuba. No obstante, en los documentos oficiales de la UE no hay indicios de que la estabilidad o la fragilidad estatal hayan influido en la decisión de adoptar una política y otra. En este sentido y teniendo en cuenta de que ambos países están considerados "países de renta media" que apenas reciben fondos de cooperación, se puede descartar que sea 
el principal factor explicativo de las diferentes políticas de la UE hacia Cuba y Venezuela.

Otra diferencia entre ambas políticas es la interlocución: mientras que en el caso de Cuba el diálogo ha sido sobre todo con el régimen, en el caso venezolano a partir de 2017 la UE ha apoyado a la oposición como contraparte. Desde 1996, la Posición Común sobre Cuba indicaba claramente el deseo de apoyar un proceso de reformas desde dentro de manera gradual y pacífica, partiendo del régimen actual. Lo mismo pasaba durante la presidencia de Hugo Chávez, cuando la UE mantuvo relaciones plenas con Venezuela, pese a algunas declaraciones críticas por la situación de derechos humanos. Fue a partir de 2013, paralelo a la transición del régimen venezolano del populismo chavista al autoritarismo competitivo, que la UE cambió su política hacia una estrategia de coerción que desde 2019 coincide con la estrategia de Washington de iniciar un cambio de régimen mediante una transición tutorizada desde el exterior. Aunque la UE mantiene su iniciativa diplomática GIC, al haber reconocido a Juan Guaidó como presidente del país, la UE no puede ni quiere mediar entre las partes. Al comparar ambas políticas, ciertamente la UE incentiva y promueve los cambios graduales que ha iniciado el régimen en Cuba y sanciona la transición inversa del gobierno de Maduro hacia el autoritarismo. Ciertamente, no es el único factor que influye en las diferentes políticas de la UE hacia ambos países, pero es un argumento explicativo de las divergencias y su evolución en la respuesta de las transiciones inversas.

\section{Referencias bibliográficas}

Alonso, J.A. y Vidal, P. (2019). La reforma económica en Cuba: atrapada en el medio. Working Paper Foro Europa-Cuba Jean Monnet Network, no. 1, Barcelona: CIDOB. Recuperado el 2 de noviembre de 2019) de http://www.foroeuropacuba.org/docs/work/working-paperno.-1.-la-reforma-econormica-en-cuba.-atrapada-en-el-medio.pdf ela? Notes Internacionals, 07/2018. Barcelona: CIDOB.

Ayuso, A. y Gratius, S. (2016). Venezuela: balas y votos: ¿cómo responder a la transición al autoritarismo caótico en Venezuela? Notes Internacionals, 07/2018. Barcelona: CIDOB.

Ayuso, A. y Gratius, S. (Eds.) (2017). Nueva etapa entre Cuba y la UE. Escenarios de futuro. Colección Monografías. Barcelona: CIDOB.

Ayuso, A. y Gratius, S. (2020). Sanciones como instrumento de coerción: ¿Cuán similares son las políticas de EE. UU. y la UE hacia Venezuela? (en proceso de revisión). América Latina Hoy, no. 85.

Börzel, T. (2002). Member States Responses to Europeanization. Journal of Common Market Studies, vol. 40, no.2, pp. 193-214.

Carcanague, S., Gómez, C. y Matelly, S. (2017). Performance des Sanctions Internationales. Paris: Institute des Relations Internationales et Stratégiques, pp. 18-19.

Carothers, T. (1999). Aiding Democracy Abroad: The Learning Curve. Washington DC: Carnegie Endowment for International Peace.
Las respuestas de la Unión Europea a las transiciones inversas en Cuba y Venezuela

Anna Ayuso

Susanne Gratius 


\begin{abstract}
Dossier América Latina: Venezuela

Carothers, T. (2020). Rejuvenating Democracy Promotion. Journal of Democracy, vol. 31, no. 1, pp. 114-123. Recuperado el 2 de noviembre de 2019 de https://muse.jhu.edu/ article/745958/pdf
\end{abstract}

Carothers, T., y Youngs, R. (2017). Democracy is Not Dying. Seeing Through the Doom and Gloom. Foreign Affairs, April, 11. Recuperado el 2 de noviembre de 2019 de https:// www.foreignaffairs.com/articles/2017-04-11/democracy-not-dying

Casado Raigón, R. (2014). Derecho Internacional. Madrid: Tecnos, $2^{\text {a }}$ edición.

Cassani, A. y Tomini, L. (2019). Post-Cold War Autocratization: Trends and Patterns of Regime Change Opposite to Democratization. Italian Political Science Review, vol. 49, no. 2, pp. 121-138.

Comisión Europea (31 de octubre de 2017). UE-Cuba: un nuevo acuerdo histórico entra en vigor el 1 de noviembre de 2017. Comunicado de prensa. Recuperado el 2 de noviembre de 2019 de https://ec.europa.eu/commission/presscorner/detail/es/IP_17_4301

Consejo de la Unión Europea (1996). 96/697/PESC: Posición común de 2 de diciembre de 1996 definida por el Consejo en virtud del artículo J.2 del Tratado de la Unión Europea, sobre Cuba. Recuperado el 2 de noviembre de 2019 de https://eur-lex.europa.eu/ LexUriServ/LexUriServ.do?uri=CELEX:31996E0697:ES:HTML

Consejo de la Unión Europea (10 de febrero de 2014). El Consejo adopta directrices para un diálogo político bilateral y un Acuerdo de Cooperación con Cuba. Comunicado de prensa 6291/14. Recuperado el 2 de noviembre de 2019 de: https://www.consilium.europa. eu/media/28988/140948.pdf

Consejo de la Unión Europea (6 de diciembre de 2016). Decisión (PESC) 2016/2233 del Consejo de 6 de diciembre de 2016 por la que se deroga la Posición Común 96/697/PESC sobre Cuba. Diario Oficial de la Unión Europea, L 337 I/41. Recuperado el 2 de noviembre de 2019 de https://eur-lex.europa.eu/legal-content/ES/TXT/PDF/?uri=CELEX:32016D2233 \&from $=$ ES

Consejo de la Unión Europea (2018). Orientaciones sobre la aplicación y evaluación de las medidas restrictivas (sanciones), 4 de mayo de 2018. Recuperado el 2 de noviembre de 2019 de https://data.consilium.europa.eu/doc/document/ST-5664-2018-INIT/es/pdf

Consejo de la Unión Europea (31 de enero de 2019). Grupo Internacional de Contacto Mandato. COLAC 12, PESC 79, Bruselas. Recuperado el 2 de noviembre de 2019 de https:// data.consilium.europa.eu/doc/document/ST-5958-2019-INIT/es/pdf

Consejo Europeo (s.f.). Sanciones: cómo y cuándo adopta la UE medidas restrictivas. Recuperado el 2 de noviembre de 2019 de https://www.consilium.europa.eu/es/policies/ sanctions/

Diamond, L. (2002). Elections Without Democracy: Thinking About Hybrid Regimes. Journal of Democracy, vol. 13, no. 2, pp. 21-35.

Diamond, L. (2015). Facing Up to the Democratic Recession. Journal of Democracy, vol. 26, no. 1, pp. 141-155. Recuperado el 2 de noviembre de 2019 de https://muse.jhu.edu/ article $/ 565645 / \mathrm{pdf}$

Doyle, M. (1997). Ways of War and Peace. New York: W.W. Norton.

Erdmann, G. (2011). Decline of Democracy: Loss of Quality, Hybridisation and Breakdown of Democracy. En G. Erdmann, M. Kneuer (Eds.), Regression of Democracy (pp. 21-58), Springer.

Freedom House (2019). Freedom in the World 2019: Democracy in Retreat. Washington DC. 
Fund for Peace (FFP) (2019). Fragile States Index Annual Report 2019. Washington DC.

Gratius, S. (2017). El papel de la Unión Europea en el triángulo Cuba, EE. UU. y Venezuela. IdeAs Idées d'Amériques 10 (États-Unis / Cuba: une nouvelle donne?), pp. 1-18.

Gratius, S. (2019). Claves de la política exterior de Cuba: presente y futuro de una revolución subsidiada, Anuario Internacional CIDOB (Perfil País: Cuba), pp. 250-255.

Gratius, S. y Puente, J.M. (2019). Las claves de la crisis venezolana, Foreign Affairs Latinoamérica, vol. 19, no. 2, pp. 5-15.

Gratius, S. y Rivero, A. (2018). Más allá del populismo de izquierda y de derecha, Revista CIDOB d'Afers Internacionals, pp. 35-61.

Hoffmann, B. (2015). The International Dimension of Authoritarian Regime Legitimation: Insights from the Cuban Case. Journal of International Relations and Development, no. 18, pp. 556-574.

Hoffmann, B. (2019). ¿Una nueva Cuba en gestación? Política y sociedad en la era post Castro", Anuario Internacional CIDOB, pp. 242-249.

Huntington, S. (1994). La tercera ola: la democratización a finales del siglo XX. BCN: Paidós Ibérica.

Leogrande, W. (2015). Cuba's Perilous Transition to the Post-Castro Era. Journal of Latin American Studies, vol. 47, no. 2, pp. 377-405.

Levitzky, S. y Way, L. (2010). Competitive Authoritarianism. Hybrid Regimes after the Cold War. Cambridge University Press.

Levitsky, S. y Ziblatt, D. (2018). How Democracies Die. New York: Crown.

Merkel, W. (2010). Systemtransformation. Eine Einführung in die Theorie und Empirie der Transformationsforschung. Berlin: Springer.

Morlino, L. (2014). Transition to Democracy: What We Know and What We Should Know. Working Paper Series 5. Rome: LUISS School of Government.

Morlino, L. (2015). Transiciones democráticas: entre cuestiones teóricas y análisis empírico. Revista Española de Ciencia Política, no. 39, pp. 17-42.

O'Donnell, G., Schmitter, P. y Whitehead, L. (1986). Transition from Authoritarian Rule: Tentative Conclusions about Uncertain Democracies. Baltimore/London: Johns Hopkins University Press.

Serbin, A. (2019). Cuba: una compleja transición. Análisis Carolina, no. 2, marzo 2019. Madrid: Fundación Carolina.

Servicio Europeo de Acción Exterior (2014). Programa Indicativo Multianual para Cuba (cooperación bilateral) 2014-2020. Recuperado el 2 de noviembre de 2019 de https:// eeas.europa.eu/headquarters/headquarters-homepage/50055/programa-indicativomultianual-para-cuba_gl

The Economist Intelligence Unit (2019). Democracy Index 2019. A year of democratic setbacks and popular protest. London: EIU. Recuperado el 2 de noviembre de 2019 de https://www.eiu.com/topic/democracy-index

Thinan, M.N. (2008). Feeling Good or Doing Good: Inefficacy of the U.S. Unilateral Sanctions Against the Military Government of Burma/Myanmar. Washington University Global Studies Law Review, vol. 7, no. 3, pp. 483-485.

Unión Europea (26 de octubre de 2012). Versión Consolidada del Tratado dela Unión Europea.
Las respuestas de la Unión Europea a las transiciones inversas en Cuba y Venezuela

Anna Ayuso

Susanne Gratius 
Dossier América Latina: Venezuela
Diario Oficial de la Unión Europea. Recuperado el 2 de noviembre de 2019 de https://eurlex.europa.eu/resource.html?uri=cellar:2bf140bf-a3f8-4ab2-b506-fd71826e6da6.0005.02/ DOC_1\&format=PDF)

U.S. Department of State (2020). Democratic Transition Framework for Venezuela. Recuperado el 2 de noviembre de 2020 de https://www.state.gov/democratic-transitionframework-for-venezuela/

Whitehead, L. (Ed.) (1996). The International Dimension of Democratization. Europe and the Americas. Oxford University Press.

Zakaria, Fareed (1997). The Rise of Illiberal Democracy. Foreign Affairs. November/ December 1997. Recuperado el 2 de noviembre de 2019 de https://www.foreignaffairs.com/ articles/1997-11-01/rise-illiberal-democracy 